\title{
Terahertz conductivity of the highly mismatched amorphous alloy, GaNBi
}

\author{
C P Vaisakh ${ }^{1}$, C T Foxon ${ }^{2}$, S V Novikov ${ }^{2}$, and R N Kini ${ }^{1}$ \\ ${ }^{1}$ Indian Institute of Science Education and Research Thiruvananthapuram \\ (IISER-TVM), CET Campus, Engineering College PO, Thiruvananthapuram, \\ Kerala, India \\ ${ }^{2}$ School of Physics and Astronomy, University of Nottingham, Nottingham \\ NG7 2RD, United Kingdom \\ E-mail: rajeevkini@iisertvm.ac.in
}

\begin{abstract}
We report terahertz optical conductivity measurements of the highly mismatched alloy, GaNBi. We find that in these amorphous GaNBi epilayers grown using plasma assisted molecular beam epitaxy, the optical conductivity is enhanced in the samples grown at higher gallium beam equivalent pressure (BEP). The optical conductivity spectra in these pseudo-amorphous epilayers follow a Drude-Smith behaviour due to charge confinement effects. The DC conductivity in the epilayers grown at the highest Ga BEP (3.1 $\times 10^{-7}$ Torr) show an increase of three orders of magnitude compared to the one grown at the lowest Ga BEP $\left(2.0 \times 10^{-7}\right.$ Torr $)$. Our measurements suggests a percolative transition from an insulating nature in the GaNBi epilayers grown at low Ga BEP to a highly conducting phase in the epilayers grown at high Ga BEP.
\end{abstract}




\section{Introduction}

The wide band gap semiconductor, Gallium Nitride $(\mathrm{GaN})$ is of great scientific as well as technological interest, due to its distinct optoelectronic properties. The material finds its applications in the manufacturing of LEDs [1, 2], Laser diodes [3], UV detectors [4], Solar cells [5], High electron mobility transistors [6] etc. GaN in its amorphous phase has potential optoelectronic applications [7, 8]. It has advantages of being less expensive and being able to be deposited on various substrates with minimal concern for lattice matching.

Semiconductors are often subjected to alloying in order to mould their properties for different applications. The process usually involves the introduction of isoelectronic elements into a semiconductor system, which have similar characteristics like atomic size, ionicity, and electronegativity [9]. Contrarily, one could often create alloy systems that contain isoelectronic species with properties very different from the host, and are usually referred to as highly mismatched alloys (HMAs) [10, 11]. The optoelectronic properties of such systems often show drastic deviation from the host semiconductor due to the strong perturbation of the band structure [11-13]. Further, the solubility limits for HMAs can be extended by growing them in the amorphous phase.

The ternary compound GaNBi is an example of a HMA, because of the very large disparity in the properties of the two isoelectronic anion species in the system namely, nitrogen and bismuth. The alloy, GaNBi differs greatly from its parent compound GaN in optoelectronic and structural characteristics. The strong shift in the optical absorption, enhancement in the conductivity and the loss of crystallinity observed in amorphous GaNBi with sort range ordering are some of the prominent differences [14-17]. 


\section{The Samples}

The amorphous GaNBi samples were grown by plasma-assisted molecular beam epitaxy (PA-MBE) on (0001) c-plane sapphire substrates. The growth technique is described in detail elsewhere $[14,15]$. We have investigated 5 samples, grown at varying gallium beam equivalent pressure (BEP) with all other MBE growth parameters kept constant. This process results in the variation of the III:V ratio $\left(\frac{G a}{N+B i}\right)$ between 0.7 to 1.3 in the amorphous GaNBi layers [15]. Here the atomic concentration of Ga divided by the sum of the atomic concentration of $\mathrm{Bi}$ and $\mathrm{N}$, was measured in GaNBi layers by Rutherford backscattering spectrometry (RBS). Samples are numbered 1 to 5 in order of increasing Ga BEP, with sample \#1 being the one grown at lowest Ga BEP of $2.0 \times 10^{-7}$ Torr and sample \#5 the one grown at the highest Ga BEP of $3.1 \times 10^{-7}$ Torr. All the samples have similar Bismuth content of around $\sim 6.5$ at. \% Bi [15]. Also all the samples were amorphous, revealing no characteristic peaks in the XRD data. The epilayers have thicknesses in the range of 0.66 to 1.00 microns. The samples grown at lower Ga BEP were seen to have unintentional oxygen incorporation as measured by RBS, which was not observed in samples grown at higher Ga BEP [15]. 


\section{The Experiment}

We have used a conventional THz-TDS system for our studies. THz radiation was generated from a biased interdigitated photoconductive antenna (iPCA) optically excited using $\sim 100 \mathrm{fs}, \mathrm{NIR}(\sim 800 \mathrm{~nm})$ pulses from a Ti-Sapphire laser. THz radiation was detected using another photoconductive antenna (PCA) with the aid of a lock-in technique. The system offers spectroscopic capabilities in the frequency range of 0.2 to $2.25 \mathrm{THz}$. The generated THz radiation was steered to the detector PCA using two pairs of off-axis parabolic mirrors. The samples were kept at the focus of the $\mathrm{THz}$ radiation created by the second parabolic mirror. The THz radiation transmitted through the sample-free area of the substrate was used as the reference. The system was purged with dry nitrogen to minimize absorption of $\mathrm{THz}$ radiation by water vapour. All measurements were done at room temperature.

\section{The Results and Discussion}

The frequency dependent complex valued $\mathrm{THz}$ refractive index and optical conductivity of all the samples were calculated from the measured THz transmittance. The THz transmittance is given by [21],

$$
\tilde{T}(\omega)=\frac{1+i(\tilde{n}(\omega)-1) \frac{\omega d}{c}}{1-i\left[\frac{\tilde{n}(\omega)-1)\left(\tilde{n}(\omega)-n_{S}\right)}{\left(n_{S}+1\right)}\right] \frac{\omega d}{c}}
$$

where, $\tilde{n}(\omega)=n+i k$ is the complex refractive index and $d$ is the thickness of the GaNBi epilayer, c is the speed of light and $n_{s}$ is the refractive index of sapphire [22]. $\widetilde{n}(\omega)$ is obtained from the measured transmittance using eqn 1 . From this the dielectric function, $\tilde{\varepsilon}(\omega)$ and optical conductivity, $\tilde{\sigma}(\omega)=\sigma_{1}+$ $i \sigma_{2}$ can be readily calculated using,

$$
\tilde{\varepsilon}(\omega)=\widetilde{n}(\omega)^{2}=\varepsilon_{G a N B i}+i \frac{\widetilde{\sigma}(\omega)}{\omega \varepsilon_{o}}
$$

The eqn 2 represents the frequency dependent complex dielectric function written as a sum of bound (first term) and free charge contributions (second term). $\varepsilon_{o}$ is the permittivity of free space and $\varepsilon_{G a N B i}$ 
is the dielectric constant of GaNBi (bound charge contribution). Since the exact value of $\varepsilon_{G a N B i}$ is not known, we have used the dielectric constant of $\mathrm{GaN}$ ( 9.4 ) [23].

The experimentally obtained $\mathrm{THz}$ optical conductivity for all the samples is shown in figure 1 . It can be seen that the optical conductivity increases with increase in Ga BEP. The THz optical conductivity does not follow a simple Drude behaviour. As an example we have shown the expected Drude spectra for sample \#3 in figure 1. Instead, the THz conductivity spectra of our GaNBi samples fit well with the Drude-Smith (DS) model [24]. The DS model have been used successfully to account for THz conductivity resulting from strong carrier backscattering/localization in a wide variety of materials ranging from conducting polymers $[25,26]$ through inorganic semiconductors $[27,28]$ to metallic thinfilms $[29,30]$. Deviation from simple Drude model have already been observed in $\mathrm{N}$ and $\mathrm{Bi}$ containing III-V alloy systems [31]. The observation of Drude-Smith nature in conductivity is a direct indication of charge confinement effects in the GaNBi alloy, which is not observed in uniform thin films or freestanding samples of GaN [20], [23], [32-34]. We will discuss the origin of the charge confinement later in this section.

The first order DS model is given by.

$$
\tilde{\sigma}_{D S}(\omega)=\frac{\varepsilon_{o} \omega_{p}^{2} \tau_{s}}{\left(1-i \omega \tau_{s}\right)} \times\left[1+\frac{C_{1}}{\left(1-i \omega \tau_{s}\right)}\right]
$$

where $v_{p}=\omega_{p} / 2 \pi$, is the plasma frequency, $\tau_{s}$ is the scattering time constant and $C_{1}$ is the localization parameter. All the DS parameters (plasma frequency, scattering time constant and localization parameter) were obtained by fitting the optical conductivity data.

As shown in figure 2(a) the scattering time constant $\left(\tau_{s}\right)$ shows a decrease with increasing Ga BEP. The $\tau_{s}$ varies from $30.8 \mathrm{fs}$ in sample \#1to $19.3 \mathrm{fs}$ in the sample \#5. It can be seen from figure 2(b) that $v_{p}$ consistently increases with increasing Ga BEP. The observed increase in plasma frequency with increasing Ga BEP is an indicator of increasing carrier density in the material. The carrier density can be obtained using the relation, $\omega_{p}=\left[n_{c} e^{2} / \varepsilon_{o} m^{*}\right]^{1 / 2}$, where $n_{c}$ and $m^{*}$ are number density and effective mass of carriers in the medium respectively. As shown in figure 2 (d), the $n_{c}$ values vary 
between $2.4 \times 10^{18} \mathrm{~cm}^{-3}$ in sample \#1 to $2.6 \times 10^{19} \mathrm{~cm}^{-3}$ in sample \#5. The effective hole mass of Gallium Nitride $\left(m^{*}=0.8 m_{e}\right)$ was used for these calculations [35].

In order to explain the increase in the carrier density we need to consider the following aspects: i) The extent of substitutional incorporation of $\mathrm{Bi}$ and, ii) The effect on the transport properties due to Bismuth incorporation. The variation of Ga BEP results in the variation of the III:V ratio in the resulting alloy. $\mathrm{N}$ rich growth conditions impede the substitutional (anionic) incorporation of $\mathrm{Bi}$ into the alloy phase. Hence a homogeneous GaNBi alloy is not formed when grown at lower Ga BEP [36, 37] A larger shift in optical absorption in the higher III: $\mathrm{V}$ ratio samples (grown at higher Ga BEP) have been reported. $[15,16]$ This is an indicator of higher extent of substitutional Bi incorporation in these samples. It has to be mentioned that though $\mathrm{Bi}$ elemental composition is similar in all the epilayers, the concentration of substitutional $\mathrm{Bi}$ is higher in epilayers grown at higher Ga BEP [16]

Generally, GaN has an unintentional $n$-doping due to nitrogen vacancies or oxygen impurities. Bismuth incorporation is shown to result in an upward shift of the valence band edge in the resulting GaNBi alloy (Valence band anticrossing model [22]). Such shifts facilitate the formation of acceptor-like native defects resulting in a $p$-type nature for the material [15]. Hence a higher bismuth incorporation is expected to result in a higher $p$-doping density. Using the 'hot-probe' method we have confirmed the $p$-type nature of our samples and we verified qualitatively that the carrier concentration increases as the Ga BEP increases. Bismuth induced acceptor states have already been observed in the HMA system, GaAsBi and also in GaSbBi using optical as well as transport measurements [38-41]. From the above facts, it can be argued that the samples grown at higher Ga BEP, have higher and more homogeneous Bismuth substitution and hence better $p$-doping. This fact directly translates to an increase in the carrier density and also the conductivity of such samples as discussed below. 
It has been shown earlier using transmission electron microscopy studies that these samples have a pseudo-amorphous structure with a high density of crystalline grains (1 - $5 \mathrm{~nm}$ diameter) of $\mathrm{GaN}$ or GaNBi embedded in an amorphous matrix [17]. Also, the crystalline grains were not found to be connected and hence the hole transport probably happens mostly through the amorphous matrix. Hence $\tau_{s}$ values represents the carrier scattering time constant in the amorphous medium. Similar semiconductor composite systems consisting of amorphous and crystal domains have been reported to show percolation transitions $[28,42,43]$. We see in figure 3 , a dependence of DC conductivity on the Ga BEP which can be well fitted by the theoretical percolation behaviour, $\sigma_{D C} \propto\left(x-x_{c}\right)^{\gamma}$, where $x$ is the Ga BEP and $x_{c}$ is the critical Ga BEP below which the conductivity is zero. Figure 3 shows the expected behaviour with the ideal value for the exponent, $\gamma=2$. This suggests that the transport in these samples occur via a percolation network of amorphous GaNBi domains.

A major factor that controls the localization effects is the probability of backscattering at the domain boundaries. This effect has to do with the height and width of the potential barrier at the boundaries. Carriers in conductive domain lying in the middle of a nonconductive medium have a high probability of backscattering and confinement. However, proximity and often interconnectedness of these conductive domains increases the odds of carriers cruising across the boundaries, reducing the 
localization effects. Such effects essentially opens up channels for long range charge transport. This leads to a percolative transition from an insulating to conductive phase.

In our GaNBi system also we see such a transition. The $C_{1}$ in sample \#1 is very close to -1 , implying heavy localization, while in sample \#5 it is close to -0.7. Hence, it can be safely argued that the GaNBi samples grown at higher Ga BEP have rather closely packed domains of $p$-type $\mathrm{GaNBi}$, distributed uniformly in the insulating matrix, compared to the samples grown under lower Ga BEP which has sparsely distributed conducting islands with no/less capability of long range transport. As a result of this, the DC conductivity of the samples grown at lowest and highest Ga BEP differ by three orders of magnitude.

\section{Conclusions}

To summarise, we have used THz-TDS and Drude-Smith analysis to shed light on transport and structural properties of the amorphous GaNBi HMA system. We were able to the estimate carrier density, mobility and DC conductivity in the amorphous GaNBi alloy. Unlike DC measurement techniques, THz measurements helps us to study the material structure on a characteristic length scale which depends on the diffusion length of charge carriers within one cycle of the $\mathrm{THz}$ electric field. Hence we were able to probe the influence of nanometer-scale domains on the carrier transport in amorphous GaNBi. We see strong carrier localization in our samples due to small size and lack of connectivity of conducting domains. A combination of higher density $p$-doping due to effective substitutional incorporation of $\mathrm{Bi}$ in the alloy and lower confinement effects due to better proximity of conductive domains, results in higher THz (and DC) conductivity in GaNBi alloys grown at higher Ga BEP. This also accounts for the percolative transition from insulating to conductive phase observed in our samples. 


\section{Acknowledgements}

The collaborative work was partially supported by a Department of Science (India) and UK-India education and research initiative (UKIERI) under the Thematic Partnerships Scheme. The MBE work at Nottingham was supported by the Engineering and Physical Sciences Research Council UK [grant numbers EP/I004203/1 and EP/K014471/1]. C.T.F. and S.V.N. would like to acknowledge Prof. K.M. Yu, Prof. W. Walukiewicz and Prof. R.W. Martin for discussions on the MBE growth of GaNBi samples.

\section{Figure Captions}

Figure 1. (a) $\sigma_{1}$, the real and (b) $\sigma_{2}$ the imaginary part of the $\mathrm{THz}$ optical conductivity obtained experimentally (symbols) for all samples (1-5) fitted with Drude-Smith Model (solid lines). The expected Drude spectra (by setting $C_{l}=0$ in eqn. (4)) for sample 3 is also plotted (dashed red line) to show the deviation of the samples from the simple Drude nature.

Figure 2. (a) The freely fit (a) scattering time constant $\left(\tau_{s}\right)$, (b) plasma frequency, $v_{p}$ and (d) Localization Parameter $\left(C_{1}\right)$ for samples grown at different Ga BEPs. We have also tried to fit the data by fixing $\tau_{s}$ values within the interval (19.3-30.8 fs) and use the other two $\left(v_{p}\right.$ and $\left.C_{1}\right)$ as free parameters. The range of values obtained for $v_{p}$ and $C_{1}$ in the above manner is indicated by the error bars in the figures. (d) The calculated carrier density, $n_{c}$ for the different samples.

Figure 3. DC conductivity $\left(\sigma_{D C}\right)$ extrapolated from Drude Smith Fit for samples grown at varying Ga BEP. The solid line is the fit using the function $\sigma_{D C} \propto(x-x c)^{2}$ 

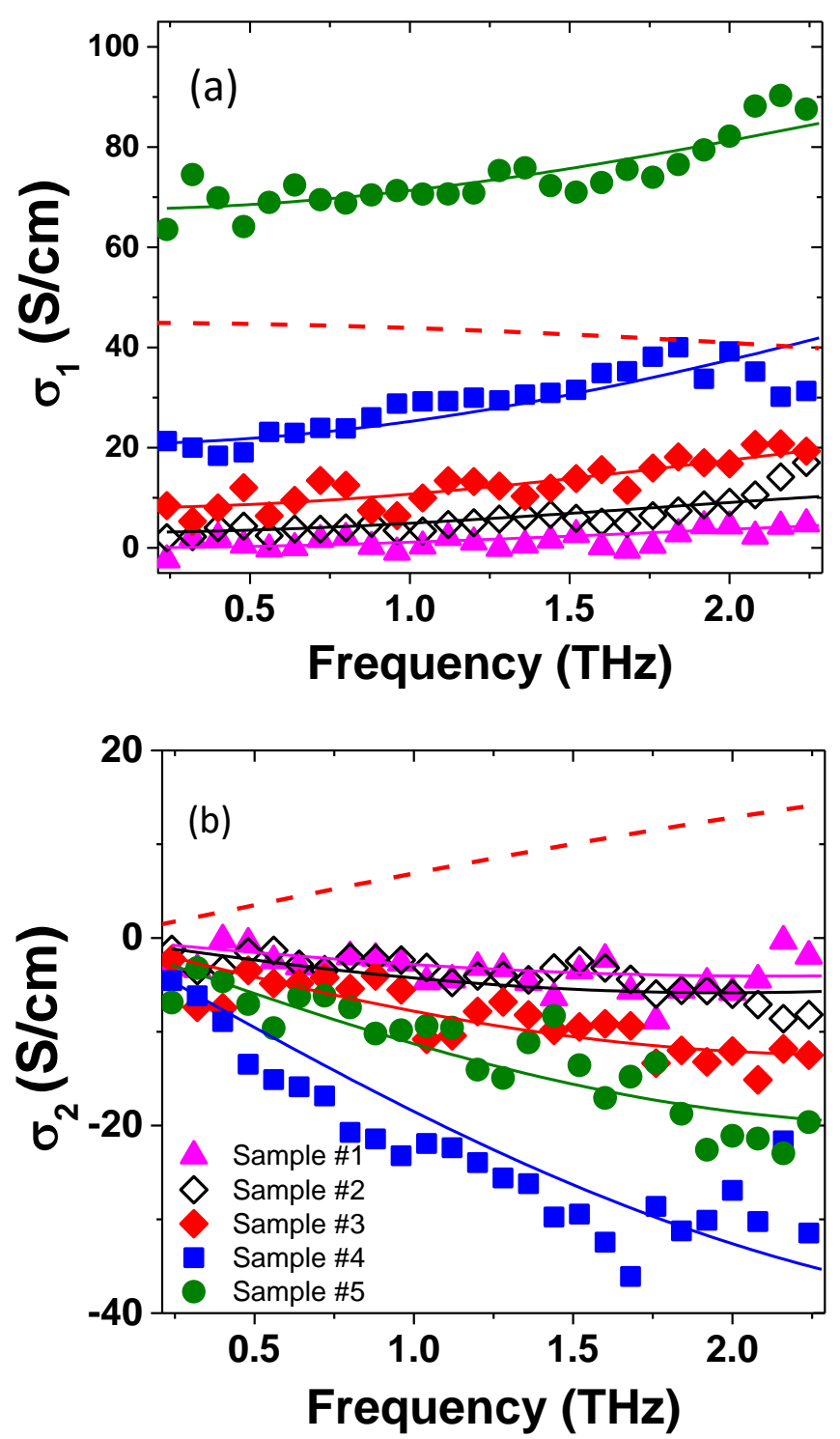

Figure 1. 

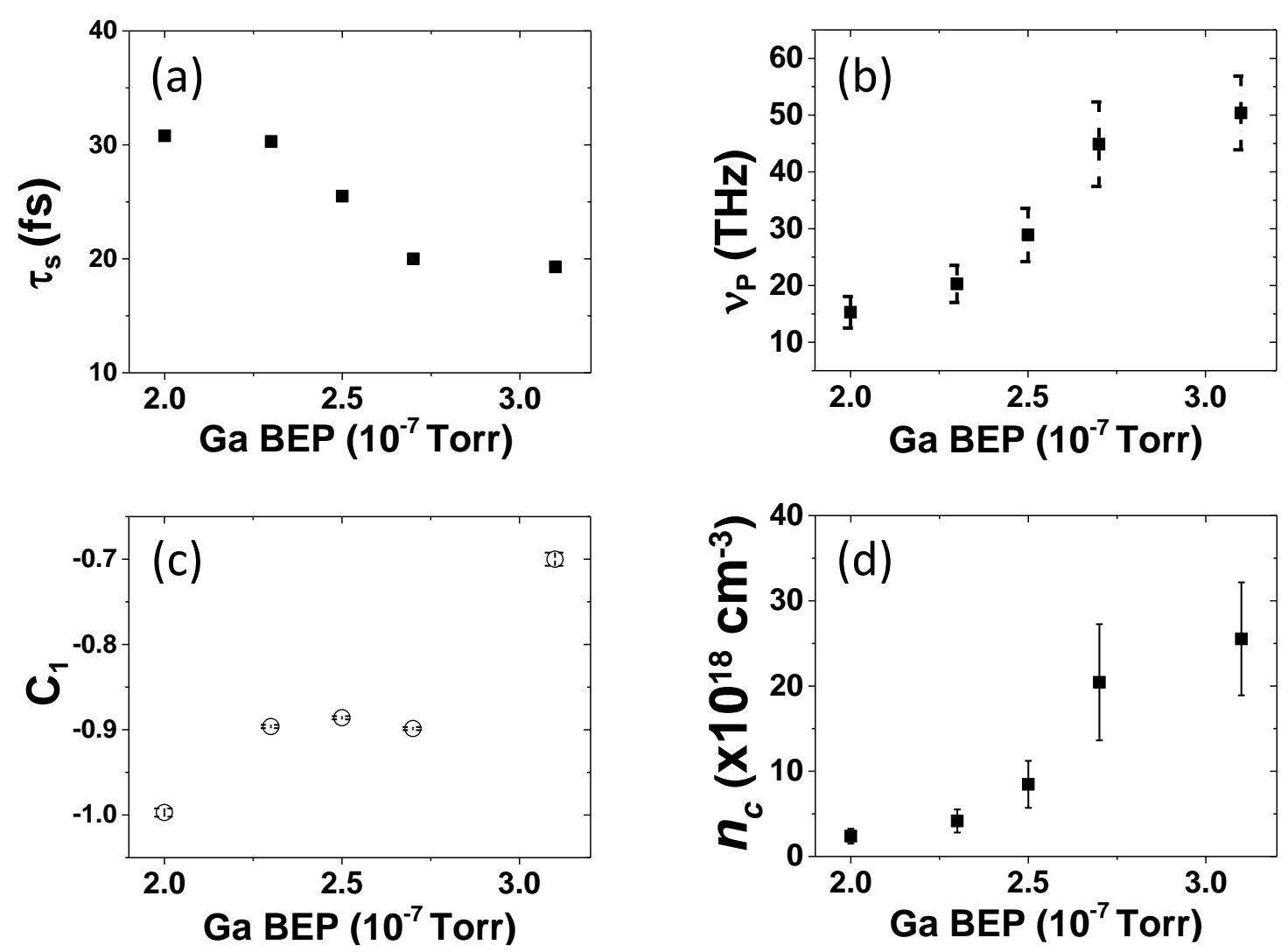

Figure 2. 


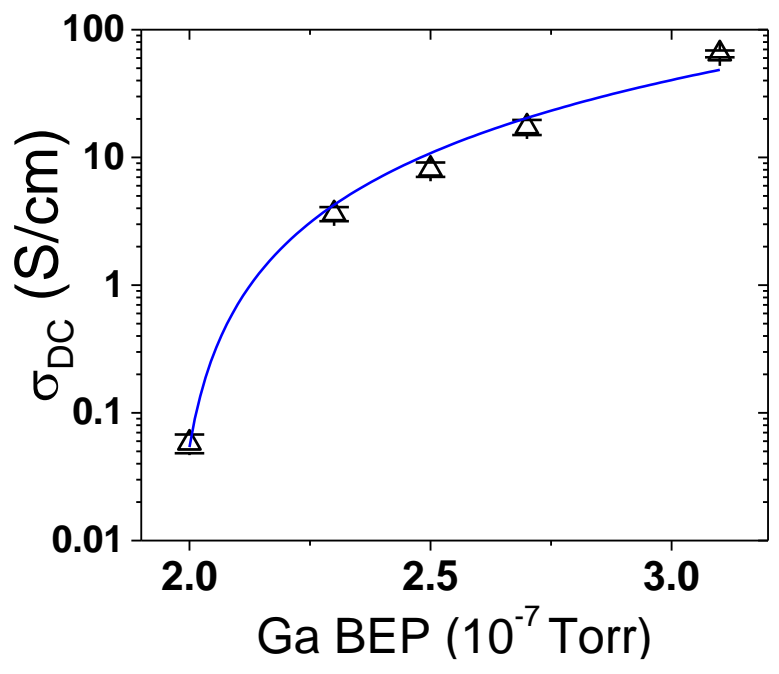

Figure 3. 
3. Miyajima T, Tojyo T, Asano T, Yanashima K, Kijima S, Hino T, et al. GaN-based blue laser diodes. J Phys Condens Matter. 2001;13:7099-114.

4. Sun X, Li D, Li Z, Song H, Jiang H, Chen Y, et al. High spectral response of self-driven GaNbased detectors by controlling the contact barrier height. Sci Rep. 2015;5:16819.

5. Bhuiyan AG, Sugita K, Hashimoto A, Yamamoto A. InGaN solar cells: Present state of the art and important challenges. IEEE J Photovoltaics. 2012;2(3):276-93.

6. Mishra UK, Shen L, Kazior TE, Wu Y. GaN-Based RF Power Devices and Amplifiers_04414367.pdf. Proc IEEE. 2008;96(2):287-305.

7. Shim SH, Shim KB, Yoon JW, Shimizu Y, Sasaki T, Koshizaki N. Blue luminescence from amorphous GaN films deposited by pulsed-laser ablation at room temperature. Thin Solid Films. 2005;472(1-2):11-5.

8. Pan X, Zhang Z, Jia L, Li H, Xie E. Room temperature visible green luminescence from aGaN:Er film deposited by DC magnetron sputtering. J Alloys Compd. 2008;458(1-2):579-82.

9. Waag A, Heinke H, Scholl S, Becker CR, Landwehr G. Growth of MgTe and Cd1-xMgxTe thin films by molecular beam epitaxy. J Cryst Growth. 1993;131(3-4):607-11.

10. Yu KM, Novikov S V., Broesler R, Demchenko IN, Denlinger JD, Liliental-Weber Z, et al. Highly mismatched crystalline and amorphous GaN1-x As x alloys in the whole composition range. J Appl Phys. 2009;106(10):103709. 
11. Alberi K, Blacksberg J, Bell LD, Nikzad S, Yu KM, Dubon OD, et al. Band anticrossing in highly mismatched Snx Ge1-x semiconducting alloys. Phys Rev B. 2008;77(7):73202.

12. Fluegel B, Francoeur S, Mascarenhas A, Tixier S, Young EC, Tiedje T. Giant spin-orbit bowing in GaAs1-xBix. Phys Rev Lett. 2006;97(6):067205.

13. Wu J, Walukiewicz W, Yu KM, Denlinger JD, Shan W, Ager JW, et al. Valence band hybridization in N-rich GaN1-xAsx alloys. Phys Rev B. 2004;70(11):115214.

14. Levander AX, Yu KM, Novikov S V., Tseng A, Foxon CT, Dubon OD, et al. GaN1-xBix: Extremely mismatched semiconductor alloys. Appl Phys Lett. 2010;97(14):141919.

15. Levander AX, Novikov S V., Liliental-Weber Z, dos Reis R, Denlinger JD, Wu J, et al. Growth and transport properties of p-type GaNBi alloys. J Mater Res. 2011;26(23):2887-94.

16. Novikov S V., Yu KM, Levander AX, Liliental-Weber Z, Dos Reis R, Kent AJ, et al. Molecular beam epitaxy of GaN 1-xBi x alloys with high bismuth content. Phys Status Solidi Appl Mater Sci. 2012;209(3):419-23.

17. Liliental-Weber Z, Dos Reis R, Levander AX, Yu KM, Walukiewicz W, Novikov S V., et al. Microstructure of GaN1-x Bi x. J Electron Mater. 2013;42(1):26-32.

18. Cocker TL, Baillie D, Buruma M, Titova L V, Sydora RD, Marsiglio F, et al. Microscopic origin of the Drude-Smith model. arxiv 1705.10350

19. Alberding BG, Thurber WR, Heilweil EJ. Direct comparison of time-resolved terahertz spectroscopy and Hall Van der Pauw methods for measurement of carrier conductivity and mobility in bulk semiconductors. J Opt Soc Am B. 2017;34(7):1392-406.

20. Engelbrecht SG, Arend TR, Zhu T, Kappers MJ, Kersting R. Terahertz electromodulation spectroscopy of electron transport in GaN. Appl Phys Lett. 2015;106(9):92107.

21. Kang TT, Yamamoto M, Tanaka M, Hashimoto A, Yamamoto A, Sudo R, et al. Terahertz characterization of semiconductor alloy AlInN: negative imaginary conductivity and its 
meaning. Opt Lett. 2009;34(16):2507-9.

22. Grischkowsky D, Keiding S, Exter M Van, Fattinger C. Far-infrared time-domain spectroscopy with terahertz beams of dielectrics and semiconductors. J Opt Soc Am B. 1990;7(10):2006-15.

23. Zhang W, Azad AK, Grischkowsky D. Terahertz studies of carrier dynamics and dielectric response of n-type, freestanding epitaxial GaN. Appl Phys Lett. 2003;82(17):2841-3.

24. Smith N. Classical generalization of the Drude formula for the optical conductivity. Phys Rev B. 2001;64(15):155106.

25. Cooke DG, Krebs FC, Jepsen PU. Direct observation of sub-100 fs mobile charge generation in a polymer-fullerene film. Phys Rev Lett. 2012;108(5):56603.

26. Unuma T, Fujii K, Kishida H, Nakamura A. Terahertz complex conductivities of carriers with partial localization in doped polythiophenes. Appl Phys Lett. 2010;97(3):33308.

27. Joyce HJ, Boland JL, Davies CL, Baig SA, Johnston MB. A review of the electrical properties of semiconductor nanowires: insights gained from terahertz conductivity spectroscopy. Semicond Sci Technol. 2016;31(10):103003.

28. Titova L V., Cocker TL, Cooke DG, Wang X, Meldrum A, Hegmann FA. Ultrafast percolative transport dynamics in silicon nanocrystal films. Phys Rev B.2011;83(8):085403.

29. Walther M, Cooke DG, Sherstan C, Hajar M, Freeman MR, Hegmann FA. Terahertz conductivity of thin gold films at the metal-insulator percolation transition. Phys Rev B. 2007;76(12):125408.

30. Kim J, Maeng I, Jung J, Song H, Son JH, Kim K, et al. Terahertz time-domain measurement of non-Drude conductivity in silver nanowire thin films for transparent electrode applications. Appl Phys Lett. 2013;102(1):011109.

31. Cooke DG, Hegmann FA, Young EC, Tiedje T. Electron mobility in dilute GaAs bismide and 
nitride alloys measured by time-resolved terahertz spectroscopy. Appl Phys Lett.

2006;89(12):122103.

32. Guo HC, Zhang XH, Liu W, Yong AM, Tang SH. Terahertz carrier dynamics and dielectric properties of GaN epilayers with different carrier concentrations. J Appl Phys. 2009;106(6):063104.

33. Tsai T-R, Chen S-J, Chang C-F, Hsu S-H, Lin T-Y, Chi C-C. Terahertz response of GaN thin films. Opt Express. 2006;14(11):4898-907.

34. Gauthier-Brun A, Teng JH, Dogheche E, Liu W, Gokarna A, Tonouchi M, et al. Properties of $\mathrm{In}_{\mathrm{x}} \mathrm{Ga}_{1-\mathrm{x}} \mathrm{N}$ films in terahertz range. Appl Phys Lett. 2012;100(7):071913.

35. Santic B. On the hole effective mass and the free hole statistics in wurtzite GaN. Semicond Sci Technol. 2003;18(4):219-24.

36. Oe K, Ando S, Sugiyama K. InSb 1-x Bi x films grown by molecular beam epitaxy. Jpn J Appl Phys. 1981;20(4):L303.

37. Yoshimoto M, Murata S, Chayahara A, Horino Y, Saraie J, Oe K. Metastable GaAsBi Alloy Grown by Molecular Beam Epitaxy. Japanese J Appl Physics. 2003;42(10 B):L1235.

38. Nargelas S, Jarašiunas K, Bertulis K, Pačebutas V. Hole diffusivity in GaAsBi alloys measured by a picosecond transient grating technique. Appl Phys Lett. 2011;98(8):082115.

39. Pettinari G, Engelkamp H, Christianen PCM, Maan JC, Polimeni A, Capizzi M, et al. Compositional evolution of Bi-induced acceptor states in GaAs 1-xBix alloy. Phys Rev B 2011;83(20):201201(R).

40. Pettinari G, Patanè A, Polimeni A, Capizzi M, Lu X, Tiedje T. Bi-induced p-type conductivity in nominally undoped Ga(AsBi). Appl Phys Lett. 2012;100(9):092109.

41. Segercrantz N, Slotte J, Makkonen I, Tuomisto F, Sandall IC, Ashwin MJ, et al. Hole density and acceptor-type defects in MBE-grown GaSb 1 - x. J Phys D Appl Phys. 2017;50:295102. 
4

5

6

7

8

9

10

11

12

13

14

15

16

17

18

19

20

21

22

23

24

25

26

27

28

29

30

31

32

33

34

35

36

37

38

39

40

41

42

43

44

45

46

47

48

49

50

51

52

53

54

55

56

57

58

59

60

42. Balberg I, Savir E, Jedrzejewski J, Nassiopoulou AG, Gardelis S. Fundamental transport processes in ensembles of silicon quantum dots. Phys Rev B. 2007;75(23):235329.

43. Dang ZM, Nan CW, Xie D, Zhang YH, Tjong SC. Dielectric behavior and dependence of percolation threshold on the conductivity of fillers in polymer-semiconductor composites. Appl Phys Lett. 2004;85(1):97-9. 